\title{
Article \\ NAPRT Expression Regulation Mechanisms: Novel Functions Predicted by a Bioinformatics Approach
}

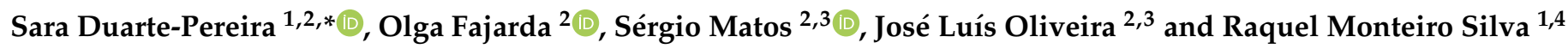 \\ 1 Department of Medical Sciences, iBiMED—Institute of Biomedicine, University of Aveiro, \\ 3810-193 Aveiro, Portugal \\ 2 IEETA-Institute of Electronics and Informatics Engineering of Aveiro, University of Aveiro, 3810-193 Aveiro, \\ Portugal; olga.oliveira@ua.pt (O.F.); aleixomatos@ua.pt (S.M.); jlo@ua.pt (J.L.O.) \\ 3 DETI-Department of Electronics, Telecommunications and Informatics, University of Aveiro, \\ 3810-193 Aveiro, Portugal \\ 4 Universidade Católica Portuguesa, Faculty of Dental Medicine, Center for Interdisciplinary Research in \\ Health (CIIS), 3504-505 Viseu, Portugal; rmsilva@ucp.pt \\ * Correspondence: sdp@ua.pt
}

Citation: Duarte-Pereira, S.; Fajarda, O.; Matos, S.; Luís Oliveira, J.; Silva, R.M. NAPRT Expression Regulation Mechanisms: Novel Functions Predicted by a Bioinformatics Approach. Genes 2021, 12, 2022. https://doi.org/10.3390/ genes12122022

Academic Editor: Anton I Petrov

Received: 15 November 2021 Accepted: 15 December 2021 Published: 20 December 2021

Publisher's Note: MDPI stays neutral with regard to jurisdictional claims in published maps and institutional affiliations.

Copyright: (c) 2021 by the authors. Licensee MDPI, Basel, Switzerland. This article is an open access article distributed under the terms and conditions of the Creative Commons Attribution (CC BY) license (https:// creativecommons.org/licenses/by/ $4.0 /)$.

\begin{abstract}
The nicotinate phosphoribosyltransferase (NAPRT) gene has gained relevance in the research of cancer therapeutic strategies due to its main role as a NAD biosynthetic enzyme. NAD metabolism is an attractive target for the development of anti-cancer therapies, given the high energy requirements of proliferating cancer cells and NAD-dependent signaling. A few studies have shown that NAPRT expression varies in different cancer types, making it imperative to assess NAPRT expression and functionality status prior to the application of therapeutic strategies targeting NAD. In addition, the recent finding of NAPRT extracellular form (eNAPRT) suggested the involvement of NAPRT in inflammation and signaling. However, the mechanisms regulating NAPRT gene expression have never been thoroughly addressed. In this study, we searched for NAPRT gene expression regulatory mechanisms in transcription factors (TFs), RNA binding proteins (RBPs) and microRNA (miRNAs) databases. We identified several potential regulators of NAPRT transcription activation, downregulation and alternative splicing and performed GO and expression analyses. The results of the functional analysis of TFs, RBPs and miRNAs suggest new, unexpected functions for the NAPRT gene in cell differentiation, development and neuronal biology.
\end{abstract}

Keywords: NAPRT (nicotinate phosphoribosyltransferase); bioinformatics; expression regulation; cell differentiation; neurodevelopment

\section{Introduction}

Nicotinate phosphoribosyltransferase (NAPRT) is an enzyme from NAD (Nicotinamide Adenine Dinucleotide) biosynthesis and is mostly studied as a cancer biomarker.

One of the cancer therapeutic strategies targeting NAD metabolism is the use of nicotinamide phosphoribosyltransferase (NAMPT) inhibitors [1-4]. NAMPT is the ratelimiting enzyme of the main NAD salvage pathway, which uses nicotinamide, while NAPRT is responsible for NAD production via the nicotinic acid precursor, known as the Preiss-Handler pathway [5,6]. In this context, NAPRT became an important biomarker for the use of nicotinic acid as a co-adjuvant in NAPRT-negative tumors [1] and for coinhibition in the cancer types that highly express NAPRT [7].

Lack of NAPRT expression was observed in several cancer types [1,8-10] and associated with NAPRT epigenetic silencing in some cases, such as gastric and lung cancer [10-13]. On the other hand, NAPRT amplifications and overexpression were reported in ovarian, breast and pancreatic cancer [7]. Differences in NAPRT expression between subtypes of cancer, namely in breast, pancreatic, lung and gastric carcinomas [7,11,14,15], suggest that individual variability should be considered in therapeutic approaches. In order to select the 
types of cancer that would better respond to NAMPT inhibition, a determination should be made on whether NAPRT is expressed and functional.

In addition to cancer, the role of NAPRT in inflammation and signaling was recently discovered and is brought by its extracellular form (eNAPRT) [16,17]. Thus, knowledge of NAPRT gene expression regulatory mechanisms under non-pathological conditions is key to understanding its biological functions and roles in disease, in addition to exploring its potential as a biomarker or therapeutic target.

We have previously studied NAPRT methylation and mutations in cancer [10,18]. In addition to promotor methylation, mutations in transcription factor binding sites (TFBS) and alternative splicing have been proposed as potential regulatory mechanisms of NAPRT gene expression $[10,11,14,18]$. As NAPRT gene expression variation is tissue-specific rather than a cancer specific alteration [15], we searched several databases of transcription factors (TFs), RNA binding proteins (RBPs) and microRNAs (miRNAs), using the NAPRT gene as a target to identify additional transcriptional and post-transcriptional mechanisms in normal conditions. We identified several potential regulators of NAPRT transcription activation, downregulation and alternative splicing. The analyses of their targets suggested unexpected functions in cell differentiation, development and neuronal biology.

\section{Methods}

\subsection{Collection of NAPRT Potential Regulators}

In order to obtain a comprehensive set of results, we searched a diverse number of databases in our approach, which are often focused on different methodologies: some collect data from experimental high throughput studies, others used computational algorithms to predict binding and others extracted data obtained directly from the literature. The databases used here are summarized in Supplementary Table S1.

\subsubsection{Transcription Factors}

In order to search for TFs that could regulate NAPRT gene expression, we surveyed one database with experimental data from the Encyclopedia of DNA Elements (ENCODE) Project [19]—Factorbook [20] — and four sources of computational predictions of transcription factor binding sites (TFBS)—UniPROBE [21]—PROMO v3.0.2, which uses the TFBS defined in the TRANSFAC database v8.3 [22,23], MotifMap [24,25] and CTCFBS v2.0 [26], using NAPRT 5'UTR sequence as an input. All unique results were considered.

\subsubsection{RNA Binding Proteins}

We collected data from a total of eight databases. Four of them included experimentally validated data-starBase v2.0, currently called The Encyclopedia of RNA Interactomes (ENCORI) [27], miRWalk v2.0 [28], CLIPdb v1.0 [29], currently integrated in POSTAR2 [30] and AURA v2.4 [31]. The other four had computationally predicted data-RBPDB v1.3 [32], RBPmap v1.0 [33], catRAPID [34] and CISBP-RNA v0.6 [35]. Starbase and miRWalk also provided information on microRNA-mRNA interactions.

In most of the queries, we searched for the entire NAPRT mRNA sequence and then performed a specific search using only $3^{\prime}$ UTR or $5^{\prime}$ UTR sequences due to their regulatory role. For some databases, such as miRWalk, the query for the gene symbol NAPRT or NAPRT1 did not retrieve any results; thus, other identifiers were used, such as EntrezID, Ensembl gene ID or UniProt accession.

\subsection{3. microRNAs}

We searched for experimentally validated data in DIANA-TarBasev7.0 [36], starBase v2.0 [27] and mirTarBase 6.0 [37]. For computationally predicted miRNA:mRNA interactions, we used DIANA-microT-CDS v5.0 [38] and TargetScan 7.0 [39]. From miRWalk v2.0 database [28], we retrieved both experimentally validated and predicted data. The best targets from the computational predictions were selected according to the following criteria: the binding site should be 8 nucleotides, and the conservation of the site sequence 
among different species should be the highest (in the case of TargetScan a conserved branch length over 0.8 for 8 nucleotides is recommended).

In addition, we obtained expression data and performed further analysis using DASHR v2.0 [40], starBase v2.0 [27], miRTarBase 6.0 [37] and miRGator v3.0 [41].

\subsection{Data Analysis}

After collecting data from the various databases (Supplementary Table S1), the results were further studied as follows (Figure 1).

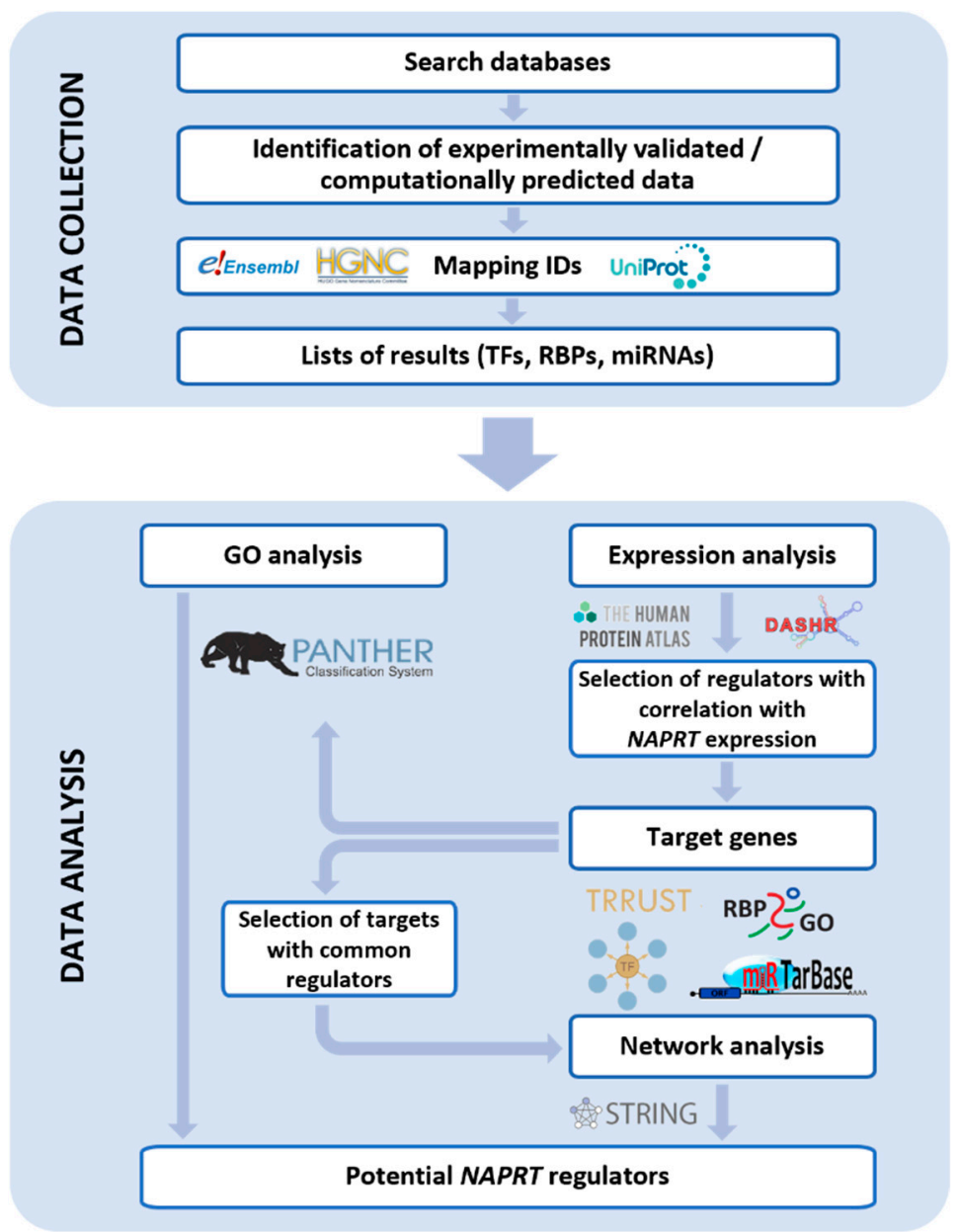

Figure 1. Data analysis. Schematic representation showing the pipeline followed to collect and analyze nicotinate phosphoribosyltransferase (NAPRT) putative binding transcription factors (TFs), RNA binding proteins (RBPs) and microRNAs (miRNAs). The list of databases can be found in Supplementary Table S1.

For TFs and RBPs, we used the HUGO Gene Nomenclature Committee (HGNC) at the European Bioinformatics Institute (www.genenames.org, accessed on 29 April 2021) and the UniProt (www.uniprot.org, accessed on 12 July 2021 [42]) databases to map each gene symbol to HUGO, Ensembl and UniProt IDs and retrieved information of the subcellular location, evidence level of expression, tissue specificity and function.

The study of gene co-expression patterns, the analysis of co-regulation networks and gene ontology enrichment analysis are commonly used methodologies to infer knowledge on gene functions. Thus, we analyzed the correlation between NAPRT gene expression and the expression of the TFs, RBPs and miRNAs from our set of results and expanded our study to the analysis of the target genes of those regulators, as described below. 


\subsubsection{Correlation with NAPRT Gene Expression}

Expression data were retrieved from the Human Protein Atlas (www.proteinatlas.org, accessed on 24 January 2021, v19.3 [43]). The dataset containing the consensus normalized expression levels summarize expression values for 62 non-pathological tissues based on transcriptomics data from three sources (Human Protein Atlas, Gene Tissue Expression (GTEx) and FANTOM5), and it was used to calculate the correlation between the expression of NAPRT gene and the identified NAPRT TFs and RBPs. In order not to assume linear relationships between co-expressed genes, Spearman's correlation was calculated. Positive correlation values above 0.5 and negative correlation values below -0.5 were considered [44]. For miRNAs, we assessed expression data from DASHR v2.0 [40] and obtained the analysis of miRNA correlation with NAPRT expression using miRGator v3.0 [41].

\subsubsection{Target Genes}

For the TFs and RBPs that presented a significant correlation with NAPRT expression, we used the TRRUST v.2 database (grnpedia.org/trrust/, accessed on 9 March 2021 [45]) and the RBP2GO database (rbp2go.dkfz.de/, accessed on 26 March 2021 [46]) to search for their target genes. The gene set enrichment analysis of the target genes of the experimentally validated miRNAs was performed with miRTarBase 6.0.

\subsubsection{Functional Analysis}

We performed a Gene Ontology (GO) analysis on the total lists of TFs, RBPs and the target genes of the expression-correlated TFs, RBPs and miRNAs using a statistical overrepresentation test (Fisher's exact test and False Discovery Rate correction) in the PANTHER Classification System (pantherdb.org, accessed on 4 October 2021, v.16.0 [47]). In the case of the TFs, we created a combined dataset to use as background in GO analysis. For that purpose, we used three sources, namely, the Human Protein Atlas [43] annotated transcription dataset (proteinatlas.org/search/protein_class\%3Atranscription+factors, accessed on 4 January 2021); the list of TFs from the ChIP-seq Peaks from the ENCODE Project [48] (encodeproject.org/, accessed on 4 January 2021, source data version: ENCODE 3 November 2018); and the human transcription factors catalogue from Lambert et al. 2018 [49]. In the case of the RBPs, we used the dataset published on the RBP2GO database [46]. On the remaining analysis, all genes in the genome were used as the enrichment background. We preferably used the PANTHER slim annotation datasets for biological processes and molecular functions, but whenever there were no statistically significant results (FDR corrected $p$ value $<0.05$ ), we chose complete annotation datasets [47].

The target genes that were common to more than one mechanism, e.g., that were targeted by at least one TF and one RBP, one TF and one miRNA or one RBP and one miRNA, were analyzed on a network of interactions obtained by using STRING (stringdb.org, accessed on 8 December 2021, v.11 [50]). We searched for data from text-mining, experiments, databases and co-expression, with a high confidence level of 0.7. Only interactions between the queried proteins were considered.

\section{Results}

\subsection{Potential Regulators of NAPRT Gene Expression}

\subsubsection{Transcription Factors}

From a total of 93 results obtained from the four databases surveyed, 80 were mapped to UniProt IDs (Supplementary Table S2). Thirty-seven of them had supporting experimental data, which derived from the ENCODE project (Supplementary Table S3). Three were common to two databases: Wilms tumor protein (WT1), Erythroid transcription factor GATA-binding factor 1 (GATA1) and YY1 transcription factor (YY1), also known as Yin and Yang 1 protein.

In order to exclude potential overrepresentation of the typical functions and biological processes in which TFs are usually involved, we used a large dataset of human TFs as background in the GO analysis. This reference dataset was compiled from three sources 
and resulted in 1849 known human TFs. In addition to the expected processes related to the transcription regulation and the involvement in the RNA metabolic process, GO analysis of the $80 \mathrm{TFs}$ revealed an overall overrepresentation for genes involved in signaling and response to stimulus (Supplementary Table S4), such as "response to UV-C" or "positive regulation of defense response to virus by host". Table 1 lists the most relevant biological processes enriched (considering a fold enrichment of over 3 with 10 or more genes).

Table 1. Biological processes overrepresentation of the 80 potential NAPRT transcription factors. Only child terms relative to 10 or more genes and with a fold enrichment of over 3 are presented, by decreasing fold-enrichment order. Complete results are listed in Supplementary Table S4.

\begin{tabular}{l}
\hline Transcrition Factors-BIOLOGICAL PROCESS \\
\hline Immune response \\
\hline Response to other organism \\
\hline Regulation of epithelial cell proliferation \\
\hline Positive regulation of immune system process \\
\hline Negative regulation of cell population proliferation \\
\hline Cellular response to growth factor stimulus \\
\hline Regulation of immune response \\
\hline Negative regulation of protein modification process \\
\hline
\end{tabular}

Considering pathway annotations (Figure 2), the highest enrichment fold was provided by three genes involved in the cadherin signaling pathway, namely, Transcription factor 7-like 2 (TCF7L2), Lymphoid enhancer binding factor (LEF1) and Transcription factor 3 (TCF3). Curiously, the set of the same three genes was responsible also for the enrichment of the Alzheimer disease presenilin pathway and the Wnt signaling pathway. In the latter, we found three additional genes: Tumor protein p53 (TP53), MYC proto-oncogene and Nuclear factor of activated T cells 1 (NFATC1). Of note, from these TFs, TCF7L2, MYC and NFATC1 have been experimentally validated.

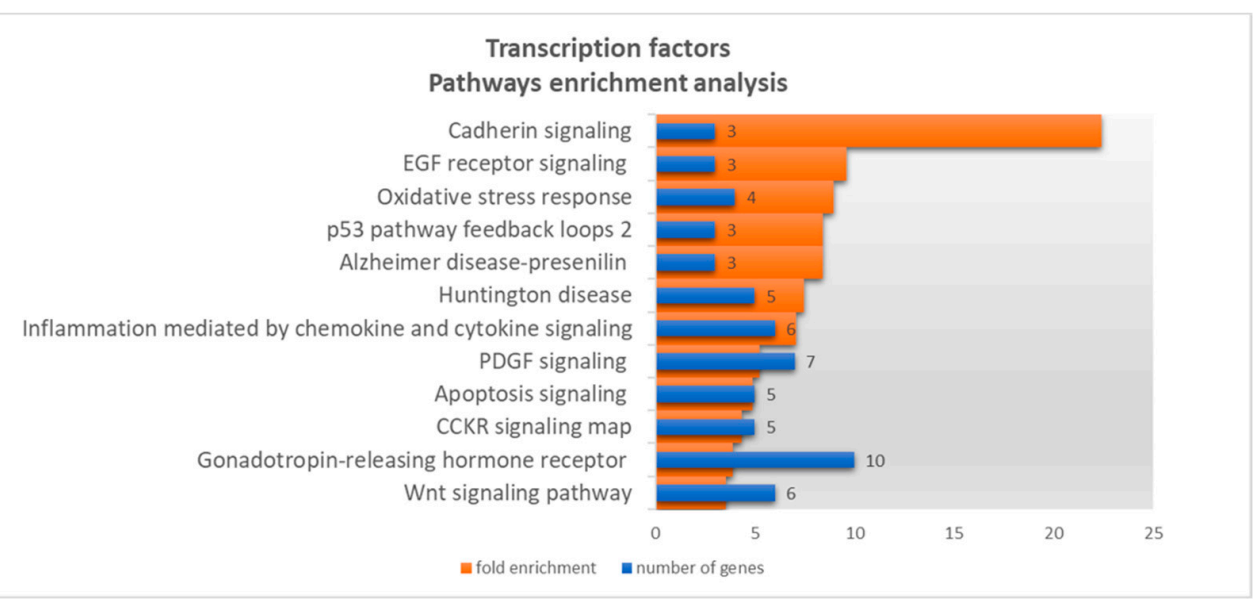

Figure 2. Gene ontology results for 80 potential NAPRT transcription factors, based on pathways annotation dataset, showing the number of genes (blue bars) and fold enrichment (orange bars) by decreasing fold enrichment order. Detailed information on GO results can be found in Supplementary Table S4. The genes responsible for the highest enrichment fold were retrieved (TCF7L2, TCF3 and LEF1).

\subsubsection{RNA Binding Proteins}

From a total of 122 RBPs (Supplementary Table S5), we obtained 113 RBPs predicted to bind NAPRT mRNA and 11 with experimentally supported data (Supplementary Table S6). 
Only splicing factor U2AF $65 \mathrm{kDa}$ subunit (U2AF2) and the FUS RNA binding protein (FUS) were both predicted and validated. Three RBPs, namely Heterogeneous nuclear ribonucleoprotein L (HNRNPL), Protein quaking (QKI) and RNA-binding motif protein 3 (RBM3), were predicted to bind specifically to NAPRT $3^{\prime} \mathrm{UTR}$; that is, no binding site was found in the remaining mRNA sequence.

We retrieved information on subcellular location, evidence level of expression, tissue specificity and function for each of the 11 experimentally validated RBPs. Several of these are reported to be functionally involved in post-transcriptional processing, as it was expected, and have ubiquitous expression. Some are necessary for normal splicing events, such as Eukaryotic initiation factor 4A-III (EIF4A3) and U2AF2, and Serine/arginine repetitive matrix protein 4 (SRRM4) is a splicing factor specifically required for neural cell differentiation.

Despite the use of a reference dataset composed by all potential human RBPs, GO analysis of the 122 NAPRT-binding RBPs still showed several processes related to splicing mechanisms, regulation of alternative splicing, splice site selection, regulation of translation and regulation of mRNA stability (Figure 3 and Supplementary Table S7). These results reflect the well-known functions of RBPs and the previously described alternative splicing events that regulate NAPRT expression [10]. Unexpectedly, several processes related to dendrite development and synapse organization were also found. The highest fold enrichment was retrieved for term regulation of dendritic spine development and axodendritic transport, associated with the functions of Fragile $\mathrm{X}$ mental retardation syndromerelated proteins 1 (FXR1) and 2 (FXR2) and Synaptic functional regulator (FMR1). Other processes related to dendrite morphogenesis and synapse organization were also enriched.

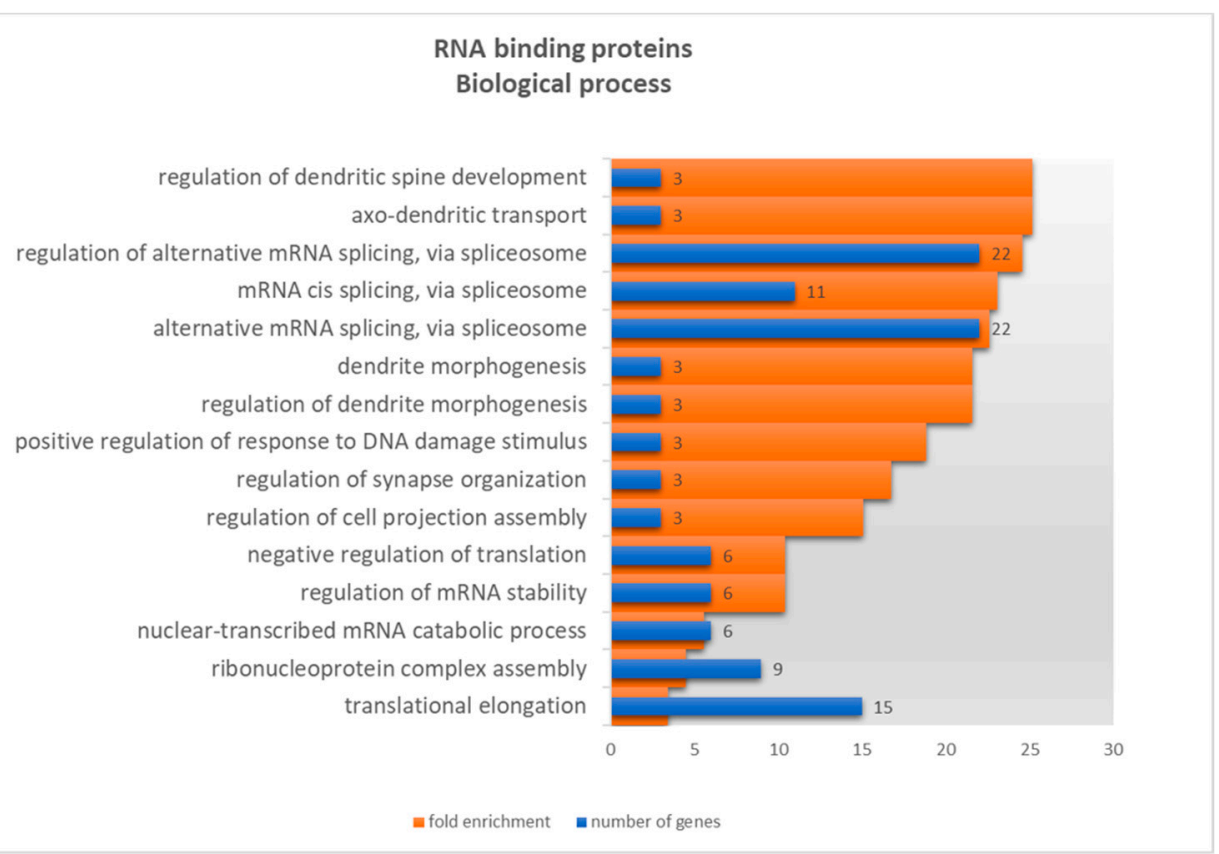

Figure 3. Gene ontology results for 122 potential NAPRT RNA binding proteins, based on biological processes annotation dataset, showing the number of genes (blue bars) and fold enrichment (orange bars) by decreasing fold enrichment order. Only child terms are presented. Detailed information on GO results can be found in Supplementary Table S7. The genes responsible for the highest enrichment fold were retrieved (FXR1, FXR2 and FMR1).

\subsection{3. microRNAs}

A total of 39 miRNAs that potentially bind NAPRT were obtained in this study (Supplementary Table S8), from which seven have experimental data (Supplementary Table S9). Two of them were obtained from two different databases (miR-218-5p and miR-92a- 
$3 p)$. From the 31 miRNAs based on computational predictions, miR-491-5p was the only prediction that followed the established criteria and was selected for further analysis, along with the seven miRNAs based on experimental data.

For eight selected miRNAs, DASHR database was used to extract data on miRNAs expression in different tissues, which can be visualized as heatmaps (Supplementary Figure S1). The results showed that most miRNAs appear to be tissue specific. For instance, the brain tissue presents the highest values for miR-197-3p, miR-218-5p, miR-491-5p and miR-92-3p (Supplementary Figure S1a-d). This is most relevant in the case of miR-491-5p (Supplementary Figure S1d), which has an overall low expression, except for brain. Of note, miR-218-5p is weakly expressed in tissues where NAPRT is strongly expressed, such as liver and blood (Supplementary Figure S1c).

\subsection{Expression Correlation between TFs, RBPs, miRNAs and the NAPRT Gene}

Spearman correlation analysis identified $11 \mathrm{TFs}$ and 9 RBPs with a significant positive correlation, from moderate to strong, with NAPRT expression, based on the Human Protein Atlas dataset of non-pathological human tissues (Table 2). With the exception of the zinc finger and BTB domain containing 7A (ZBTB7A), which acts as a repressor, all these TFs can both activate or repress their target genes. Most of the RBPs are involved in splicing or in the general process of transcription, and many of them are a part of the hnRNP family. The zinc finger CCHC-type containing 17 (ZCCHC17) was the only RBP that presented a significant negative correlation with NAPRT expression.

Table 2. Transcription factors and RNA binding proteins with a significant correlation with NAPRT expression.

\begin{tabular}{|c|c|c|c|c|}
\hline & Gene Symbol & Protein Name & Spearman & $p$ Value \\
\hline \multirow{11}{*}{ 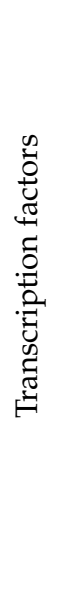 } & BCL3 & BCL3 transcription coactivator & 0.725 & $2.64 \times 10^{-11}$ \\
\hline & CEBPB & CCAAT enhancer binding protein $\beta$ & 0.618 & $8.62 \times 10^{-8}$ \\
\hline & JUN & Jun proto-oncogene, AP-1 transcription factor subunit & 0.532 & $8.46 \times 10^{-6}$ \\
\hline & MAFB & MAF bZIP transcription factor B & 0.569 & $1.42 \times 10^{-6}$ \\
\hline & PML & PML nuclear body scaffold & 0.568 & $1.44 \times 10^{-6}$ \\
\hline & RXRA & retinoid $X$ receptor $\alpha$ & 0.628 & $4.67 \times 10^{-8}$ \\
\hline & STAT6 & signal transducer and activator of transcription 6 & 0.573 & $1.14 \times 10^{-6}$ \\
\hline & TCF3 & transcription factor 3 & 0.543 & $5.23 \times 10^{-6}$ \\
\hline & TMEM37 & transmembrane protein 37 & 0.566 & $1.65 \times 10^{-6}$ \\
\hline & YY1 & YY1 transcription factor & 0.522 & $1.33 \times 10^{-5}$ \\
\hline & ZBTB7A & zinc finger and BTB domain containing 7A & 0.590 & $4.52 \times 10^{-7}$ \\
\hline \multirow{10}{*}{ 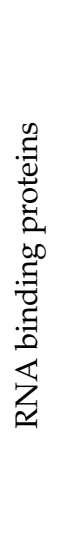 } & ESRP2 & epithelial splicing regulatory protein 2 & 0.532 & $8.72 \times 10^{-6}$ \\
\hline & HNRNPAB & heterogeneous nuclear ribonucleoprotein A/B & 0.638 & $2.41 \times 10^{-8}$ \\
\hline & HNRNPH1 & heterogeneous nuclear ribonucleoprotein H1 & 0.503 & $3.15 \times 10^{-5}$ \\
\hline & HNRNPL & heterogeneous nuclear ribonucleoprotein L & 0.589 & $4.76 \times 10^{-7}$ \\
\hline & PCBP1 & poly $(\mathrm{rC})$ binding protein 1 & 0.522 & $1.34 \times 10^{-5}$ \\
\hline & PTBP1 & polypyrimidine tract binding protein 1 & 0.608 & $1.63 \times 10^{-7}$ \\
\hline & РTBP3 & polypyrimidine tract binding protein 3 & 0.627 & $5.02 \times 10^{-8}$ \\
\hline & SRSF2 & serine and arginine rich splicing factor 2 & 0.621 & $7.30 \times 10^{-8}$ \\
\hline & YBX1 & Y-box binding protein 1 & 0.523 & $1.32 \times 10^{-5}$ \\
\hline & ZCCHC17 & zinc finger CCHC-type containing 17 & -0.636 & $2.82 \times 10^{-8}$ \\
\hline
\end{tabular}


We also found a significant correlation between miR-92a-3p and miR-218-5p and NAPRT expression in different datasets (Table 3). Interestingly, miR-92a-3p had a positive or a negative correlation depending on tissues. The strongest correlation was positive and was found in the dataset of differentiated embryonic stem cells.

Table 3. Significant correlations between NAPRT and miRNA expression.

\begin{tabular}{|c|c|c|c|c|}
\hline & & Dataset & $\begin{array}{l}\text { Spearman } \\
\text { Correlation }\end{array}$ & $p$-Value \\
\hline \multirow{5}{*}{ hsa-miR-92a-3p } & GSE34608 & Pulmonary tuberculosis and sarcoidosis & -0.797 & $1.3 \times 10^{-5}$ \\
\hline & GSE38974 & Chronic obstructive pulmonary disease & -0.626 & $4.1 \times 10^{-4}$ \\
\hline & GSE42095 & Differentiated embryonic stem cells & 0.830 & $4.8 \times 10^{-7}$ \\
\hline & GSE28544 & Breast cancer & 0.710 & $5.1 \times 10^{-5}$ \\
\hline & GSE15076 & Monocyte-derived dendritic cells & 0.600 & $4.4 \times 10^{-2}$ \\
\hline hsa-miR-218-5p & GSE38226 & Liver fibrosis & -0.726 & $9.7 \times 10^{-5}$ \\
\hline
\end{tabular}

\subsection{Analysis of Target Genes}

Next, we identified 340 known targets for the 11 TFs that correlate with NAPRT expression (Supplementary Table S10). There were no reported targets only for the transmembrane protein 37 (TMEM37). Forty-three target genes were regulated by two or more TFs. For the nine RBPs positively correlated with NAPRT expression, we obtained 148 target genes, and one more for the negatively correlated RBP (Supplementary Table S11). Fifty-five of them were targeted by two or more RBPs. Among them, we found many heterogeneous nuclear ribonucleoproteins (hnRNPs), small nuclear ribonucleoproteins (snRNPs) and serine/arginine-rich splicing factors (SRSFs).

In miRTarBase, we performed Gene Set Enrichment analysis for all target genes of a given miRNA. For each of the eight miRNAs studied, we selected the genes with at least two validation methods, resulting in 1 gene for miR-1915-3p, 63 genes for miR-197-3p, 37 genes for miR-218-5p, 8 genes for miR-491-5p and 51 genes for miR-92a-3p. Between them, only the TP53 gene was targeted by miR-92 and miR-491. The total list of 158 miRNA targets is found on Supplementary Table S12).

Excluding duplicates, we obtained a total of 626 target genes.

\subsubsection{Gene Ontology}

We performed GO on the collection of all target genes, including the targets of the $11 \mathrm{TFs}$, the targets of the nine RBPs positively correlated with NAPRT expression and the RBP negatively correlated with NAPRT expression and the targets of the four miRNAs for which we found validated results in a total of 626 genes. Table 4 lists the most relevant biological processes enriched (fold enrichment of over 3 with 10 or more genes), and the complete results are found in Supplementary Table S13. The highest number of genes, 32 , was mapped to the inflammation mediated by chemokine and cytokine signaling pathway, but several signaling pathways appeared, including cholecystokinin receptor (CCKR), gonadotropin-releasing hormone receptor (GnRHR), Toll receptor and interleukin pathways. A few key cellular pathways related to both proliferation and apoptosis were also enriched, such as p53, Ras and TGF- $\beta$ signaling. In addition, we found Alzheimer's disease and Huntington disease pathways, blood coagulation and angiogenesis within the most relevant results (considering a fold enrichment of above 3, with 10 or more genes). 
Table 4. Pathway overrepresentation of 626 targets of TFs and RBPs with a correlation with NAPRT expression and miRNA targets. Only the child terms relative to 10 or more genes and with a fold enrichment of over 3 are presented by decreasing fold enrichment order. Complete results are in Supplementary Table S13.

\begin{tabular}{l}
\hline Target Genes-PATHWAYS \\
\hline p53 pathway \\
\hline Toll receptor signaling pathway \\
\hline Apoptosis signaling pathway \\
\hline Interleukin signaling pathway \\
\hline Transcription regulation by bZIP transcription factor \\
\hline Blood coagulation \\
\hline CCKR signaling map \\
\hline Ras Pathway \\
\hline Gonadotropin-releasing hormone receptor pathway \\
\hline Alzheimer disease-presenilin pathway \\
\hline Inflammation mediated by chemokine and cytokine signaling pathway \\
\hline TGF- $\beta$ signaling pathway \\
\hline Angiogenesis \\
\hline Huntington disease
\end{tabular}

For miRNAs, a separate GO analysis of each set of targets provided significant results only for miR-218 and miR-92. The terms with the highest fold enrichment were all related to differentiation or development. The terms with the highest number of genes were not only mostly related to the regulation of transcription but also proliferation and differentiation terms (Supplementary Table S14). Overall, GO analysis revealed an overrepresentation for genes involved in developmental processes, mostly of cardiovascular and nervous systems. Genes related to cell differentiation and proliferation, mainly of nervous system and epithelium, were also enriched. In addition, we found several processes related to signaling, including the Wnt pathway, cell adhesion and cell death.

\subsubsection{Targets Co-Regulated}

We identified 20 genes that were targeted by at least two different mechanisms within our set of results, e.g., a pair of TF/RBP, TF/miRNA or RBP/miRNA. The unique gene found within the target genes of the three types of regulators was cadherin 1 (CDH1).

In order to find potential associations between these 20 target genes, we used the STRING platform to obtain an interaction network (Figure 4). For 3 out of the 20 genes, no interactions were found (ABCC3, IL1R1 and ZNF175). We retrieved 30 interactions (edges) between 17 genes (nodes), with an average degree of 3 and a significant protein-protein interaction enrichment $p$-value $\left(1.45 \times 10^{-12}\right)$. Eleven out of the twenty proteins were found to have two or more interactions, TP53 being the one with the highest number of interactions (nine). 


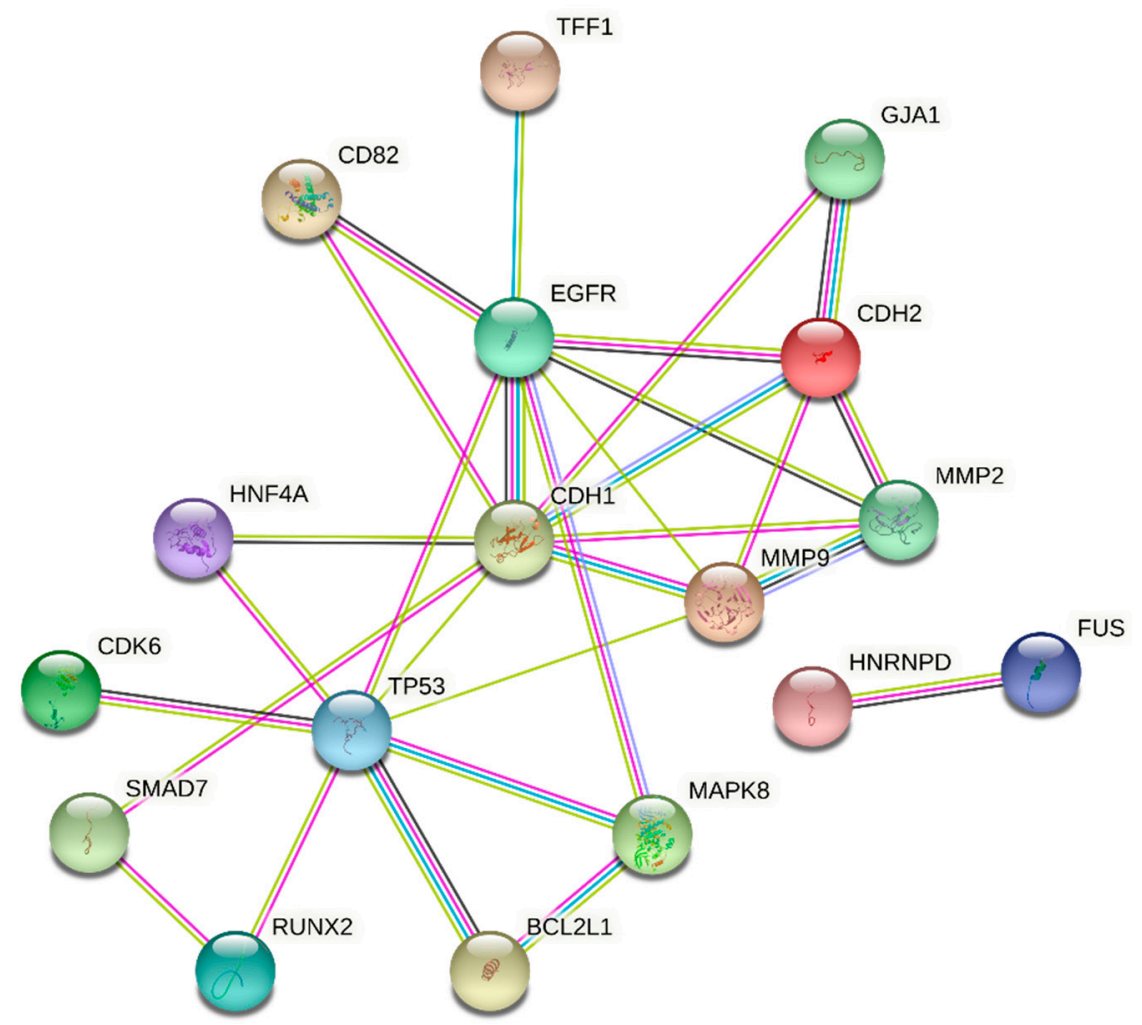

Figure 4. Network of interactions between 17 genes targeted by more than one NAPRT potential regulator. The network was obtained by using STRING (string-db.org). The line color of the edges indicates the type of interaction evidence: experimentally determined (pink), from curated databases (light blue), text mining (light green), co-expression (dark grey) and protein homology (purple). The color of the nodes is arbitrary.

\section{Discussion}

The goal of this study was to investigate the mechanisms responsible for the regulation of NAPRT expression. A thorough survey resulted in identifying a group of TFs, RBPs and miRNAs that potentially bind to the NAPRT gene or mRNA, regulating its expression at transcriptional and post-transcriptional levels. For an interaction to occur, the target mRNA and the protein (in the case of TFs and RBPs) or the miRNA must be expressed in the same tissue, which resulted in additional gene expression and network analyses. Based on the rationale of co-regulatory networks of gene expression [51], co-regulated genes are genes that are regulated by at least one common mechanism and are likely to participate in similar biological functions. Tissue expression specificity can also provide important insights into function. Herein, we directed the study to a normal, physiological and nonpathological context. Surprisingly, functional analysis of NAPRT regulators uncovered a link with developmental processes, cell differentiation and cell proliferation, which are common to the three mechanisms (TFs, RBPs and miRNAs). In addition, we found that the Cadherin signaling, which was previously associated to NAPRT [14], also correlated to NAPRT expression regulators.

Among our results, we found several general regulators, such as MYC and TP53, which are two ubiquitous TFs that are known to activate the transcription of growth-related genes and are involved in cell proliferation/apoptotic regulation processes. In the set of RBPs, we could find numerous heterogeneous nuclear ribonucleoproteins (hnRNPs), serine/arginine (SR) proteins and RNA binding motif (RBM) proteins, which are major families of splicing factors. NAPRT gene has a high number of alternatively spliced transcripts, which are probably tissue specific, mostly in the brain [10]. This could explain the high number of RBPs involved in splicing that were found to potentially bind NAPRT. 
Additionally, the GO analysis of TFs showed an enrichment in cadherin signaling and in immune response, as several processes relate to viral response and interleukin production, and the GO analysis of the RBPs suggest an association to dendrite development and synapse organization. In more detail, the most relevant genes found in these analyses were the TFs TCF7L2, TCF3 and LEF1; and RBPs FXR1, FXR2 and FMR1, as these were responsible for the enrichment of the processes with the highest fold. TCF3 is involved in lymphocyte development, and TCF7L2 has been implicated in neural development and diseases [52], such as multiple sclerosis, where a role in demyelination and remyelination has been suggested [53]. LEF1 is specifically expressed in lymphoid tissues, and it activates transcription in the presence of $\beta$-catenin (CTNNB1). They participate in the Wnt signaling pathway, which is further discussed below. FXR1, FXR2 and FMR1 belong to the Fragile X family of RBPs. Their role in neurogenesis has been reviewed elsewhere [54]. In addition to Fragile X Syndrome, they have been associated with other neurological disorders and cancer [55].

Correlation analysis with NAPRT gene expression revealed eleven TFs and ten RBPs, which would have a greater probability of exerting a regulatory mechanism in NAPRT transcription activation and processing (Table 2). RBP ZCCHC17, which has unique negative correlation with NAPRT expression, has been scarcely studied, but the most relevant studies place it as a regulator in Alzheimer's disease [56]. Of note, signaling pathways related to Alzheimer's and Huntington's diseases, both degenerative brain diseases, also appeared in the GO analysis.

Among the positively NAPRT correlated factors, only TFs BCL3, PML and YY1 were identified in experimentally validated data. $B C L 3$ gene encodes for a transcription coactivator, previously known as B-Cell Lymphoma 3-Encoded Protein, due to its role in lymphoma and leukemia. It was initially implicated in cell lineage determination [57] and more recently associated with Wnt/ $\beta$-catenin signaling in other types of cancer [58]. A positively correlated RBP was the epithelial splicing regulatory protein 2 (ESRP2), which particularly regulates the splicing of transcripts that undergo changes in splicing during epithelial-to-mesenchymal transition (EMT), namely CD44 and CTNND1. Among the TFs correlated with NAPRT expression, ZBTB7A (Zinc Finger and BTB Domain Containing 7A) was the only TF that acts only as a repressor (and not activator), according to the TRRUST database. It represses SLC2A3 (GLUT3), a glucose membrane transporter, which is mostly known for its specific expression in neurons. Very recently, GLUT3's role in glioblastoma has been described [59].

GO analysis results of the target genes of the miRNAs miR-218-5p and miR-92a-3p are consistent with the results for TFs and RBPs, where an enrichment in neural and cardiac development and cell differentiation was found, and a considerable number of processes related to signaling included the Wnt pathway.

Although most research studies on these microRNAs are related to cancer, where they have been identified as tumor suppressors, other areas are being investigated. MiR-218 was described as motor-neuron specific and its downregulation was associated with neurodegeneration in mice [60] and amyotrophic lateral sclerosis in humans [61]. In association with the Wnt/b-catenin pathway, miR-218 is involved in osteogenic differentiation [62], in neuronal differentiation of adipose stem cells [63] and, more recently, it was shown that miR-218 is required for functional neural-like cells [64]. MiR-92 is also involved in processes of EMT, particularly related to angiogenesis [65]. Moreover, miR-491 is associated with the post-transcriptional regulation of dopamine transporters in neural cells [66]. In addition, miR-491 is among the miRNAs identified as regulators of the transcription factor TCF7, which acts in the Wnt/b-catenin pathway [67]. Considering their expression patterns, we suggest that both miR-218 and miR-491 might have a role in NAPRT downregulation in the brain.

Most of the mentioned TFs, RBPs and miRNAs are also associated with cancer development. One particular pathway is the Wnt signaling pathway, mostly via the b-catenin pathway (also known as the canonical pathway), which has functions in tissue develop- 
ment and homeostasis, and is often altered in cancer (reviewed in [68]). Cellular processes such as proliferation, differentiation, adhesion and survival take place upon Wnt activation.

One of the cellular processes where the Wnt signaling pathway is determinant is the epithelial to mesenchymal transition (EMT), which is a key step not only in embryonic development but also in carcinogenesis. In the EMT, epithelial cells with strong cell-cell adhesion are converted in mesenchymal cells, which present more motile and invasive properties. After the discovery that NAPRT expression was lost in several EMT-subtypes of gastric tumors, Lee et al. suggested that NAPRT is involved in the Wnt pathway and plays a putative role in the stabilization of the $\beta$-catenin destruction complex [14]. In their study, a positive correlation between the expression of NAPRT and E-cadherin was described. In order to strengthen this relation, in our study, the global analysis of all targets showed that the $\mathrm{CDH} 1$ protein was targeted by three types of NAPRT expression regulators, namely by TCF3 (a TF), ESRP2 (an RBP) and the miR-92. These three potential NAPRT regulators were found to be significantly correlated with NAPRT expression. In the canonical Wnt signaling pathway, activation/repression of the target genes depends on the TCF/LEF family of TFs, which comprises TCF3, TCF7L2 and LEF1, which bind to b-catenin. TCF7L2 binding to NAPRT was retrieved from experimental data and TCF3 presented a positive correlation with NAPRT expression, further supporting NAPRT functions in this signaling pathway.

Our GO results also suggested a role for NAPRT in immune signaling, consistent with recent findings that the extracellular NAPRT protein (eNAPRT) triggered inflammatory responses in macrophages, mostly from the NF-kB pathway, and enhanced macrophage differentiation from circulating monocytes $[16,17]$. This is relevant, as it supports the new NAPRT roles independent of its enzymatic function on NAD production.

\section{Conclusions}

Our study suggests that NAPRT is involved in differentiation and developmental processes and showed that, beyond its application in cancer therapeutic strategies involving NAMPT, NAPRT may participate in the process of carcinogenesis and tumor progression. Particularly in neural development, our results are supported by the established association of NAPRT mutations with neurological/neurodevelopmental diseases, namely, attention-deficit/hyperactivity disorder and schizophrenia [69,70]. Studies in zebrafish also suggested that partial loss of function of the NAPRT gene results in abnormal brain development [70].

As such, our results indicate NAPRT potential regulators that should be experimentally studied. This particularly includes TFs TCF3/TCF7L2, the lesser known RBP ZCCHC17 and the miRNAs miR-218 and miR-491. The previously described link of NAPRT with the Wnt signaling [14] was emphasized by several of our analyses. Thus, given the role of this pathway in differentiation and developmental processes, NAPRT should also be further explored in this context.

Supplementary Materials: The following are available online at https://www.mdpi.com/article/10 .3390/genes12122022/s1, Figure S1: Expression heatmaps for the experimentally validated miRNAs, obtained from DASHR (http:/ / dashr2.lisanwanglab.org/, accessed on 14 December 2021). Table S1: Databases used for data collection on transcription factors, RNA binding proteins and microRNAs, Table S2: Full list of potential NAPRT transcription factors (total of 80), Table S3: Transcription factors with experimentally supporting data of NAPRT binding, Table S4: Gene ontology results for transcription factors, Table S5: Full list of potential NAPRT RNA binding proteins (total of 122), Table S6: RNA binding proteins with experimentally supporting data of NAPRT binding, Table S7: Gene ontology results for RNA binding proteins, Table S8: Full list of potential NAPRT microRNAs (total of 39), Table S9: microRNAs with experimentally supporting data of NAPRT binding, Table S10: Target genes of the TFs significantly correlated with NAPRT expression (total of 340), Table S11: Target genes of the RBPs significantly correlated with NAPRT expression (total of 149), Table S12: Target genes of the miRNAs experimentally validated (total of 158), Table S13: Gene ontology results of the target genes (total of 626) from the transcription factors and the RNA binding proteins that 
have a significant correlation with NAPRT expression and the microRNAs. Biological process (A) and molecular function (B) from the PANTHER slim annotations datasets, PANTHER pathways (C), by decreasing fold enrichment order. Only child terms are presented, Table S14: Top GO results for miR-92 (A) and miR-218 (B) target genes. The processes with a fold enrichment over 100 and relative to more than 10 genes are shown. Only child terms are presented.

Author Contributions: Conceptualization, S.D.-P. and R.M.S.; methodology, S.D.-P.; software, S.M.; validation, S.D.-P., J.L.O. and R.M.S.; formal analysis, S.D.-P. and O.F.; investigation, S.D.-P.; resources, J.L.O.; data curation, S.D.-P. and S.M.; writing_original draft preparation, S.D.-P.; writing—review and editing, S.D.-P., O.F., J.L.O. and R.M.S.; visualization, S.D.-P. and S.M.; supervision, J.L.O. and R.M.S.; project administration, R.M.S.; funding acquisition, J.L.O. All authors have read and agreed to the published version of the manuscript.

Funding: This research was funded by Fundação para a Ciência e Tecnologia: SFRH/BD/108890/2015; Fundação para a Ciência e Tecnologia: UIDB/04501/2020 (POCI-01-0145-FEDER-007628); Fundação para a Ciência e Tecnologia: UIDB/00127/2020; Fundação para a Ciência e Tecnologia: $\mathrm{UIDB} / 04279 / 2020$.

Institutional Review Board Statement: Not applicable.

Informed Consent Statement: Not applicable.

Data Availability Statement: Not applicable.

Acknowledgments: This work was supported by FEDER (Programa Operacional Factores de Competitividade-COMPETE) and by FCT (Fundação para a Ciência e Tecnologia), within the grant SFRH-BD-108890-2015 to SDP, and the projects UIDB/04501/2020 (POCI-01-0145-FEDER-007628) to iBiMED, UIDB/00127/2020 to IEETA and UIDB/04279/2020 to CIIS. We thank FCT/MCTES and UCP for CEEC institutional funding of RMS.

Conflicts of Interest: The authors declare no conflict of interest.

\section{References}

1. Watson, M.; Roulston, A.; Bélec, L.; Billot, X.; Marcellus, R.; Bédard, D.; Bernier, C.; Branchaud, S.; Chan, H.; Dairi, K.; et al. The small molecule GMX1778 is a potent inhibitor of NAD+ biosynthesis: Strategy for enhanced therapy in nicotinic acid phosphoribosyltransferase 1-deficient tumors. Mol. Cell Biol. 2009, 29, 5872-5888. [CrossRef] [PubMed]

2. Tarrant, J.M.; Dhawan, P.; Singh, J.; Zabka, T.S.; Clarke, E.; DosSantos, G.; Dragovich, P.S.; Sampath, D.; Lin, T.; McCray, B.; et al. Preclinical models of nicotinamide phosphoribosyltransferase inhibitor-mediated hematotoxicity and mitigation by co-treatment with nicotinic acid. Toxicol. Mech. Methods 2015, 25, 201-211. [CrossRef]

3. Cole, J.; Guiot, M.C.; Gravel, M.; Bernier, C.; Shore, G.C.; Roulston, A. Novel NAPRT specific antibody identifies small cell lung cancer and neuronal cancers as promising clinical indications for a NAMPT inhibitor/niacin co-administration strategy. Oncotarget 2017, 8, 77846-77859. [CrossRef]

4. Zhao, G.; Green, C.F.; Hui, Y.H.; Prieto, L.; Shepard, R.; Dong, S.; Wang, T.; Tan, B.; Gong, X.; Kays, L.; et al. Discovery of a Highly Selective NAMPT Inhibitor That Demonstrates Robust Efficacy and Improved Retinal Toxicity with Nicotinic Acid Coadministration. Mol. Cancer Ther. 2017, 16, 2677-2688. [CrossRef]

5. Rongvaux, A.; Andris, F.; Van Gool, F.; Leo, O. Reconstructing eukaryotic NAD metabolism. Bioessays 2003, 25, 683-690. [CrossRef]

6. Magni, G.; Amici, A.; Emanuelli, M.; Orsomando, G.; Raffaelli, N.; Ruggieri, S. Enzymology of NAD+ homeostasis in man. Cell Mol. Life Sci. 2004, 61, 19-34. [CrossRef]

7. Piacente, F.; Caffa, I.; Ravera, S.; Sociali, G.; Passalacqua, M.; Vellone, V.G.; Becherini, P.; Reverberi, D.; Monacelli, F.; Ballestrero, A.; et al. Nicotinic Acid Phosphoribosyltransferase Regulates Cancer Cell Metabolism, Susceptibility to NAMPT Inhibitors, and DNA Repair. Cancer Res. 2017, 77, 3857-3869. [CrossRef]

8. Olesen, U.H.; Hastrup, N.; Sehested, M. Expression patterns of nicotinamide phosphoribosyltransferase and nicotinic acid phosphoribosyltransferase in human malignant lymphomas. APMIS 2011, 119, 296-303. [CrossRef] [PubMed]

9. O'Brien, T.; Oeh, J.; Xiao, Y.; Liang, X.; Vanderbilt, A.; Qin, A.; Yang, L.; Lee, L.B.; Ly, J.; Cosino, E.; et al. Supplementation of nicotinic acid with NAMPT inhibitors results in loss of in vivo efficacy in NAPRT1-deficient tumor models. Neoplasia 2013, 15, 1314-1329. [CrossRef] [PubMed]

10. Duarte-Pereira, S.; Pereira-Castro, I.; Silva, S.S.; Correia, M.G.; Neto, C.; da Costa, L.T.; Amorim, A.; Silva, R.M. Extensive regulation of nicotinate phosphoribosyltransferase (NAPRT) expression in human tissues and tumors. Oncotarget 2016, 7, 1973-1983. [CrossRef] [PubMed]

11. Shames, D.S.; Elkins, K.; Walter, K.; Holcomb, T.; Du, P.; Mohl, D.; Xiao, Y.; Pham, T.; Haverty, P.M.; Liederer, B.; et al. Loss of NAPRT1 expression by tumor-specific promoter methylation provides a novel predictive biomarker for NAMPT inhibitors. Clin. Cancer Res. 2013, 19, 6912-6923. [CrossRef] [PubMed] 
12. Peterse, E.F.P.; van den Akker, B.E.W.M.; Niessen, B.; Oosting, J.; Suijker, J.; de Jong, Y.; Danen, E.H.J.; Cleton-Jansen, A.M.; Bovée, J.V.M.G. NAD Synthesis Pathway Interference Is a Viable Therapeutic Strategy for Chondrosarcoma. Mol. Cancer Res. 2017, 15, 1714-1721. [CrossRef] [PubMed]

13. Fons, N.R.; Sundaram, R.K.; Breuer, G.A.; Peng, S.; McLean, R.L.; Kalathil, A.N.; Schmidt, M.S.; Carvalho, D.M.; Mackay, A.; Jones, C.; et al. PPM1D mutations silence NAPRT gene expression and confer NAMPT inhibitor sensitivity in glioma. Nat. Commun. 2019, 10, 3790. [CrossRef] [PubMed]

14. Lee, J.; Kim, H.; Lee, J.E.; Shin, S.J.; Oh, S.; Kwon, G.; Choi, Y.Y.; White, M.A.; Paik, S.; Cheong, J.H.; et al. Selective Cytotoxicity of the NAMPT Inhibitor FK866 Toward Gastric Cancer Cells With Markers of the Epithelial-Mesenchymal Transition, Due to Loss of NAPRT. Gastroenterology 2018, 155, 799-814.e13. [CrossRef]

15. Chowdhry, S.; Zanca, C.; Rajkumar, U.; Koga, T.; Diao, Y.; Raviram, R.; Liu, F.; Turner, K.; Yang, H.; Brunk, E.; et al. NAD metabolic dependency in cancer is shaped by gene amplification and enhancer remodelling. Nature 2019, 569, 570-575. [CrossRef]

16. Managò, A.; Audrito, V.; Mazzola, F.; Sorci, L.; Gaudino, F.; Gizzi, K.; Vitale, N.; Incarnato, D.; Minazzato, G.; Ianniello, A.; et al. Extracellular nicotinate phosphoribosyltransferase binds Toll like receptor 4 and mediates inflammation. Nat. Commun. 2019, 10, 4116. [CrossRef]

17. Audrito, V.; Messana, V.G.; Deaglio, S. NAMPT and NAPRT: Two Metabolic Enzymes With Key Roles in Inflammation. Front. Oncol. 2020, 10, 358. [CrossRef]

18. Duarte-Pereira, S.; Silva, S.S.; Azevedo, L.; Castro, L.; Amorim, A.; Silva, R.M. NAMPT and NAPRT1: Novel polymorphisms and distribution of variants between normal tissues and tumor samples. Sci. Rep. 2014, 4, 6311. [CrossRef]

19. Davis, C.A.; Hitz, B.C.; Sloan, C.A.; Chan, E.T.; Davidson, J.M.; Gabdank, I.; Hilton, J.A.; Jain, K.; Baymuradov, U.K.; Narayanan, A.K.; et al. The Encyclopedia of DNA elements (ENCODE): Data portal update. Nucleic Acids Res. 2018, 46, D794-D801. [CrossRef]

20. Wang, J.; Zhuang, J.; Iyer, S.; Lin, X.Y.; Greven, M.C.; Kim, B.H.; Moore, J.; Pierce, B.G.; Dong, X.; Virgil, D.; et al. Factorbook.org: A Wiki-based database for transcription factor-binding data generated by the ENCODE consortium. Nucleic Acids Res. 2013, 41, D171-D176. [CrossRef]

21. Hume, M.A.; Barrera, L.A.; Gisselbrecht, S.S.; Bulyk, M.L. UniPROBE, update 2015: New tools and content for the online database of protein-binding microarray data on protein-DNA interactions. Nucleic Acids Res. 2015, 43, D117-D122. [CrossRef]

22. Farré, D.; Roset, R.; Huerta, M.; Adsuara, J.E.; Roselló, L.; Albà, M.M.; Messeguer, X. Identification of patterns in biological sequences at the ALGGEN server: PROMO and MALGEN. Nucleic Acids Res. 2003, 31, 3651-3653. [CrossRef]

23. Messeguer, X.; Escudero, R.; Farré, D.; Núñez, O.; Martínez, J.; Albà, M.M. PROMO: Detection of known transcription regulatory elements using species-tailored searches. Bioinformatics 2002, 18, 333-334. [CrossRef]

24. Daily, K.; Patel, V.R.; Rigor, P.; Xie, X.; Baldi, P. MotifMap: Integrative genome-wide maps of regulatory motif sites for model species. BMC Bioinform. 2011, 12, 495. [CrossRef] [PubMed]

25. Xie, X.; Rigor, P.; Baldi, P. MotifMap: A human genome-wide map of candidate regulatory motif sites. Bioinformatics 2009, 25, 167-174. [CrossRef]

26. Ziebarth, J.D.; Bhattacharya, A.; Cui, Y. CTCFBSDB 2.0: A database for CTCF-binding sites and genome organization. Nucleic Acids Res. 2013, 41, D188-D194. [CrossRef]

27. Li, J.H.; Liu, S.; Zhou, H.; Qu, L.H.; Yang, J.H. starBase v2.0: Decoding miRNA-ceRNA, miRNA-ncRNA and protein-RNA interaction networks from large-scale CLIP-Seq data. Nucleic Acids Res. 2014, 42, D92-D97. [CrossRef]

28. Dweep, H.; Gretz, N. miRWalk2.0: A comprehensive atlas of microRNA-target interactions. Nat. Methods 2015, 12, 697. [CrossRef] [PubMed]

29. Yang, Y.C.; Di, C.; Hu, B.; Zhou, M.; Liu, Y.; Song, N.; Li, Y.; Umetsu, J.; Lu, Z.J. CLIPdb: A CLIP-seq database for protein-RNA interactions. BMC Genom. 2015, 16, 51. [CrossRef]

30. Zhu, Y.; Xu, G.; Yang, Y.T.; Xu, Z.; Chen, X.; Shi, B.; Xie, D.; Lu, Z.J.; Wang, P. POSTAR2: Deciphering the post-transcriptional regulatory logics. Nucleic Acids Res. 2019, 47, D203-D211. [CrossRef] [PubMed]

31. Dassi, E.; Re, A.; Leo, S.; Tebaldi, T.; Pasini, L.; Peroni, D.; Quattrone, A. AURA 2: Empowering discovery of post-transcriptional networks. Translation 2014, 2, e27738. [CrossRef]

32. Cook, K.B.; Kazan, H.; Zuberi, K.; Morris, Q.; Hughes, T.R. RBPDB: A database of RNA-binding specificities. Nucleic Acids Res. 2011, 39, D301-D308. [CrossRef]

33. Paz, I.; Kosti, I.; Ares, M.; Cline, M.; Mandel-Gutfreund, Y. RBPmap: A web server for mapping binding sites of RNA-binding proteins. Nucleic Acids Res. 2014, 42, W361-W367. [CrossRef] [PubMed]

34. Agostini, F.; Zanzoni, A.; Klus, P.; Marchese, D.; Cirillo, D.; Tartaglia, G.G. catRAPID omics: A web server for large-scale prediction of protein-RNA interactions. Bioinformatics 2013, 29, 2928-2930. [CrossRef]

35. Ray, D.; Kazan, H.; Cook, K.B.; Weirauch, M.T.; Najafabadi, H.S.; Li, X.; Gueroussov, S.; Albu, M.; Zheng, H.; Yang, A.; et al. A compendium of RNA-binding motifs for decoding gene regulation. Nature 2013, 499, 172-177. [CrossRef]

36. Vlachos, I.S.; Paraskevopoulou, M.D.; Karagkouni, D.; Georgakilas, G.; Vergoulis, T.; Kanellos, I.; Anastasopoulos, I.L.; Maniou, S.; Karathanou, K.; Kalfakakou, D.; et al. DIANA-TarBase v7.0: Indexing more than half a million experimentally supported miRNA:mRNA interactions. Nucleic Acids Res. 2015, 43, D153-D159. [CrossRef] [PubMed]

37. Chou, C.H.; Chang, N.W.; Shrestha, S.; Hsu, S.D.; Lin, Y.L.; Lee, W.H.; Yang, C.D.; Hong, H.C.; Wei, T.Y.; Tu, S.J.; et al. miRTarBase 2016: Updates to the experimentally validated miRNA-target interactions database. Nucleic Acids Res. 2016, 44, D239-D247. [CrossRef] 
38. Paraskevopoulou, M.D.; Georgakilas, G.; Kostoulas, N.; Vlachos, I.S.; Vergoulis, T.; Reczko, M.; Filippidis, C.; Dalamagas, T.; Hatzigeorgiou, A.G. DIANA-microT web server v5.0: Service integration into miRNA functional analysis workflows. Nucleic Acids Res. 2013, 41, W169-W173. [CrossRef] [PubMed]

39. Agarwal, V.; Bell, G.W.; Nam, J.W.; Bartel, D.P. Predicting effective microRNA target sites in mammalian mRNAs. eLife 2015, 4, 5005. [CrossRef]

40. Kuksa, P.P.; Amlie-Wolf, A.; Katanić, Ž.; Valladares, O.; Wang, L.S.; Leung, Y.Y. DASHR 2.0: Integrated database of human small non-coding RNA genes and mature products. Bioinformatics 2019, 35, 1033-1039. [CrossRef] [PubMed]

41. Cho, S.; Jang, I.; Jun, Y.; Yoon, S.; Ko, M.; Kwon, Y.; Choi, I.; Chang, H.; Ryu, D.; Lee, B.; et al. MiRGator v3.0: A microRNA portal for deep sequencing, expression profiling and mRNA targeting. Nucleic Acids Res. 2013, 41, D252-D257. [CrossRef] [PubMed]

42. Consortium, U. UniProt: A worldwide hub of protein knowledge. Nucleic Acids Res. 2019, 47, D506-D515. [CrossRef] [PubMed]

43. Uhlén, M.; Fagerberg, L.; Hallström, B.M.; Lindskog, C.; Oksvold, P.; Mardinoglu, A.; Sivertsson, Å.; Kampf, C.; Sjöstedt, E.; Asplund, A.; et al. Proteomics. Tissue-based map of the human proteome. Science 2015, 347, 1260419. [CrossRef]

44. Akoglu, H. User's guide to correlation coefficients. Turk. J. Emerg. Med. 2018, 18, 91-93. [CrossRef]

45. Han, H.; Cho, J.W.; Lee, S.; Yun, A.; Kim, H.; Bae, D.; Yang, S.; Kim, C.Y.; Lee, M.; Kim, E.; et al. TRRUST v2: An expanded reference database of human and mouse transcriptional regulatory interactions. Nucleic Acids Res. 2018, 46, D380-D386. [CrossRef]

46. Caudron-Herger, M.; Jansen, R.E.; Wassmer, E.; Diederichs, S. RBP2GO: A comprehensive pan-species database on RNA-binding proteins, their interactions and functions. Nucleic Acids Res. 2021, 49, D425-D436. [CrossRef]

47. Mi, H.; Ebert, D.; Muruganujan, A.; Mills, C.; Albou, L.P.; Mushayamaha, T.; Thomas, P.D. PANTHER version 16: A revised family classification, tree-based classification tool, enhancer regions and extensive API. Nucleic Acids Res. 2021, 49, D394-D403. [CrossRef] [PubMed]

48. Consortium, E.P. An integrated encyclopedia of DNA elements in the human genome. Nature 2012, 489, 57-74. [CrossRef] [PubMed]

49. Lambert, S.A.; Jolma, A.; Campitelli, L.F.; Das, P.K.; Yin, Y.; Albu, M.; Chen, X.; Taipale, J.; Hughes, T.R.; Weirauch, M.T. The Human Transcription Factors. Cell 2018, 172, 650-665. [CrossRef]

50. Szklarczyk, D.; Gable, A.L.; Lyon, D.; Junge, A.; Wyder, S.; Huerta-Cepas, J.; Simonovic, M.; Doncheva, N.T.; Morris, J.H.; Bork, P.; et al. STRING v11: Protein-protein association networks with increased coverage, supporting functional discovery in genome-wide experimental datasets. Nucleic Acids Res. 2019, 47, D607-D613. [CrossRef]

51. Allocco, D.J.; Kohane, I.S.; Butte, A.J. Quantifying the relationship between co-expression, co-regulation and gene function. BMC Bioinform. 2004, 5, 18. [CrossRef]

52. Bem, J.; Brożko, N.; Chakraborty, C.; Lipiec, M.A.; Koziński, K.; Nagalski, A.; Szewczyk, Ł.; Wiśniewska, M.B. Wnt/ ß-catenin signaling in brain development and mental disorders: Keeping TCF7L2 in mind. FEBS Lett. 2019, 593, 1654-1674. [CrossRef] [PubMed]

53. Vallée, A.; Vallée, J.N.; Guillevin, R.; Lecarpentier, Y. Interactions Between the Canonical WNT/Beta-Catenin Pathway and PPAR Gamma on Neuroinflammation, Demyelination, and Remyelination in Multiple Sclerosis. Cell Mol. Neurobiol. 2018, 38, 783-795. [CrossRef] [PubMed]

54. Patzlaff, N.E.; Shen, M.; Zhao, X. Regulation of Adult Neurogenesis by the Fragile X Family of RNA Binding Proteins. Brain Plast. 2018, 3, 205-223. [CrossRef]

55. Majumder, M.; Johnson, R.H.; Palanisamy, V. Fragile X-related protein family: A double-edged sword in neurodevelopmental disorders and cancer. Crit. Rev. Biochem. Mol. Biol. 2020, 55, 409-424. [CrossRef] [PubMed]

56. Tomljanovic, Z.; Patel, M.; Shin, W.; Califano, A.; Teich, A.F. ZCCHC17 is a master regulator of synaptic gene expression in Alzheimer's disease. Bioinformatics 2018, 34, 367-371. [CrossRef]

57. Ohno, H.; Takimoto, G.; McKeithan, T.W. The candidate proto-oncogene bcl-3 is related to genes implicated in cell lineage determination and cell cycle control. Cell 1990, 60, 991-997. [CrossRef]

58. Legge, D.N.; Shephard, A.P.; Collard, T.J.; Greenhough, A.; Chambers, A.C.; Clarkson, R.W.; Paraskeva, C.; Williams, A.C. BCL-3 promotes a cancer stem cell phenotype by enhancing $\beta$-catenin signalling in colorectal tumour cells. Dis. Model Mech. 2019, 12, 37697. [CrossRef]

59. Libby, C.J.; Gc, S.; Benavides, G.A.; Fisher, J.L.; Williford, S.E.; Zhang, S.; Tran, A.N.; Gordon, E.R.; Jones, A.B.; Tuy, K.; et al. A role for GLUT3 in glioblastoma cell invasion that is not recapitulated by GLUT1. Cell Adhes. Migr. 2021, 15, 101-115. [CrossRef]

60. Amin, N.D.; Bai, G.; Klug, J.R.; Bonanomi, D.; Pankratz, M.T.; Gifford, W.D.; Hinckley, C.A.; Sternfeld, M.J.; Driscoll, S.P.; Dominguez, B.; et al. Loss of motoneuron-specific microRNA-218 causes systemic neuromuscular failure. Science 2015, 350, 1525-1529. [CrossRef] [PubMed]

61. Reichenstein, I.; Eitan, C.; Diaz-Garcia, S.; Haim, G.; Magen, I.; Siany, A.; Hoye, M.L.; Rivkin, N.; Olender, T.; Toth, B.; et al. Human genetics and neuropathology suggest a link between miR-218 and amyotrophic lateral sclerosis pathophysiology. Sci. Transl. Med. 2019, 11, 5264. [CrossRef] [PubMed]

62. Zhang, W.B.; Zhong, W.J.; Wang, L. A signal-amplification circuit between miR-218 and Wnt/ $\beta$-catenin signal promotes human adipose tissue-derived stem cells osteogenic differentiation. Bone 2014, 58, 59-66. [CrossRef] [PubMed]

63. Hu, F.; Sun, B.; Xu, P.; Zhu, Y.; Meng, X.H.; Teng, G.J.; Xiao, Z.D. MiR-218 Induces Neuronal Differentiation of ASCs in a Temporally Sequential Manner with Fibroblast Growth Factor by Regulation of the Wnt Signaling Pathway. Sci. Rep. 2017, 7, 39427. [CrossRef] 
64. Khalil, W.; Tiraihi, T.; Soleimani, M.; Baheiraei, N.; Zibara, K. Conversion of Neural Stem Cells into Functional Neuron-Like Cells by MicroRNA-218: Differential Expression of Functionality Genes. Neurotox. Res. 2020, 38, 707-722. [CrossRef] [PubMed]

65. Yamada, N.O.; Heishima, K.; Akao, Y.; Senda, T. Extracellular Vesicles Containing MicroRNA-92a-3p Facilitate Partial EndothelialMesenchymal Transition and Angiogenesis in Endothelial Cells. Int. J. Mol. Sci. 2019, 20, 4406. [CrossRef]

66. Jia, X.; Wang, F.; Han, Y.; Geng, X.; Li, M.; Shi, Y.; Lu, L.; Chen, Y. miR-137 and miR-491 Negatively Regulate Dopamine Transporter Expression and Function in Neural Cells. Neurosci. Bull. 2016, 32, 512-522. [CrossRef]

67. Hrdličková, R.; Nehyba, J.; Bargmann, W.; Bose, H.R. Multiple tumor suppressor microRNAs regulate telomerase and TCF7, an important transcriptional regulator of the Wnt pathway. PLoS ONE 2014, 9, e86990. [CrossRef]

68. Wiese, K.E.; Nusse, R.; van Amerongen, R. Wnt signalling: Conquering complexity. Development 2018, 145, 165902. [CrossRef]

69. Lasky-Su, J.; Neale, B.M.; Franke, B.; Anney, R.J.; Zhou, K.; Maller, J.B.; Vasquez, A.A.; Chen, W.; Asherson, P.; Buitelaar, J.; et al. Genome-wide association scan of quantitative traits for attention deficit hyperactivity disorder identifies novel associations and confirms candidate gene associations. Am. J. Med. Genet. B Neuropsychiatr. Genet. 2008, 147B, 1345-1354. [CrossRef]

70. Periyasamy, S.; John, S.; Padmavati, R.; Rajendren, P.; Thirunavukkarasu, P.; Gratten, J.; Vinkhuyzen, A.; McRae, A.; Holliday, E.G.; Nyholt, D.R.; et al. Association of Schizophrenia Risk With Disordered Niacin Metabolism in an Indian Genome-wide Association Study. JAMA Psychiatry 2019, 2019, 1335. [CrossRef] 